\title{
Surface heat flux determination using a genetic algorithm
}

\author{
L. P. Kanevce ${ }^{1}$, G. H. Kanevce ${ }^{2}$ \& V. B. Mitrevski ${ }^{1}$ \\ ${ }^{I}$ Faculty of Technical Sciences, St. Kliment Ohridski University, \\ Macedonia \\ ${ }^{2}$ Macedonian Academy of Sciences and Arts, Macedonia
}

\begin{abstract}
This paper deals with surface heat flux estimation on the basis of transient temperature measurements using inverse method. As a direct problem the transient heat conduction through a plane slab isolated at one boundary and exposed to the heat flux on the other side is considered. The numerical experiments have been conducted in order to simulate real measurements. The inverse problem consists of heat flux estimation on the basis of experimental temperature response. The objective function, which has to be minimized in order to estimate unknown heat flux, is the least square function of experimental and calculated temperature data. A variant of genetic algorithm has been used for minimization of the objective function. Different convergence histories are presented, compared and discussed. Also, comparison between estimated heat flux change and exact solution and comparison between calculated and experimental transient temperature response are presented.
\end{abstract}

Keywords: genetic algorithm, heat flux, inverse problems, parameter estimation.

\section{Introduction}

An inverse approach to parameter estimation in the last few decades has become widely used in various scientific disciplines: like mechanical engineering, heat and mass transfer, fluid mechanics, optimization of the processes, optimal shape defining etc. By inverse method, on the basis of known effects, causes have to be defined, in contrast to the standard, direct method when causes are known and effects have to be determined. Application of inverse method is especially important in investigation of the processes where necessary direct measurements 
are difficult or impracticable and also in finding the optimum regarding different aspects. In fact, the values of parameters which figures in the model of a physical process have to be obtained from the observed data.

Inverse problems of heat and mass transfer are based on temperature and/or heat flux measurements in order to obtain unknown parameter values appearing in the mathematical formulation of a considered problem. This approach is applied in problems of conduction, convection, radiation, and their combination like melting and solidification, in problems of heat and mass transfer with phase change, in drying processes, in composite materials exposed to high temperatures and so on [1]. In this work, the problem of heat flux evaluation on the free surface of the heated body on the basis of transient temperature measurements in the body is analyzed.

\section{The objective function}

For the parameter estimation problem the objective function is defined as the sum of the squared errors between experimental data and corresponding model predicted values $[2,3]$ :

$$
\mathrm{E}(\mathbf{P})=[\mathbf{Y}-\mathbf{T}(\mathbf{P})]^{\mathrm{T}}[\mathbf{Y}-\mathbf{T}(\mathbf{P})]
$$

where $\mathbf{Y}^{\mathrm{T}}=\left[\mathrm{Y}_{1}, \mathrm{Y}_{2}, \ldots, \mathrm{Y}_{\mathrm{imax}}\right]$ is a vector of measured temperatures, $\mathbf{T}^{\mathrm{T}}=\left[\mathrm{T}_{1}(\mathbf{P})\right.$, $\left.\mathrm{T}_{2}(\mathbf{P}), \ldots \mathrm{T}_{\text {imax }}(\mathbf{P})\right]$ calculated temperatures at times $\tau_{\mathrm{i}}(\mathrm{i}=1,2, \ldots, \operatorname{imax}), \mathbf{P}^{\mathrm{T}}=$ $\left[\mathrm{P}_{1}, \mathrm{P}_{2}, \ldots, \mathrm{P}_{\mathrm{N}}\right]$ vector of unknown parameters, imax total number of measured temperatures, $\mathrm{N}$ total number of unknown parameters and superscript $\mathrm{T}$ indicates transpose. The parameter estimation problem is solved by minimization of function E. Parameter values at the minimum are solution of this problem. In this paper, for minimization in such a way defined objective function the genetic algorithm has been used.

\section{Genetic algorithm}

The genetic algorithm [4-6] is categorized as a random search method. During performing calculations the generator of random numbers is called several thousand times. However, the method also contains the certain rules which systematically lead toward solution. Because of the random search characteristic the method has slow convergence but in the same time with its reproduction and selection rules it is stable and reliable.

There are certain variations of this method depending on the application. The basic algorithm, fig. 1, processes the following characteristics:

- An initial population of a given number of candidates is selected by random choice.

- The fitness of each of the members of the population is determined using performance measure for the problem. 
- The members of current generation of the population reproduce to create the next generation. The choice of the parents is random but the reproduction should favor the members with better fitness. During reproduction, crossover of the genes results in new members (children) not originally in the previous generation but related to them. If the child is better than the worst of the previous generation it survives and become the member of the new generation.

- With mutation probability some of the children mutate. These mutations introduce new characteristics not in the previous generation and not directly related to the previous generation but they may result in a more suitable child. Mutated child survives if it is better than the worst of the previous generation.

- The process of reproduction repeats until obtaining the member with desired characteristics or until a preset number of generations have been created.

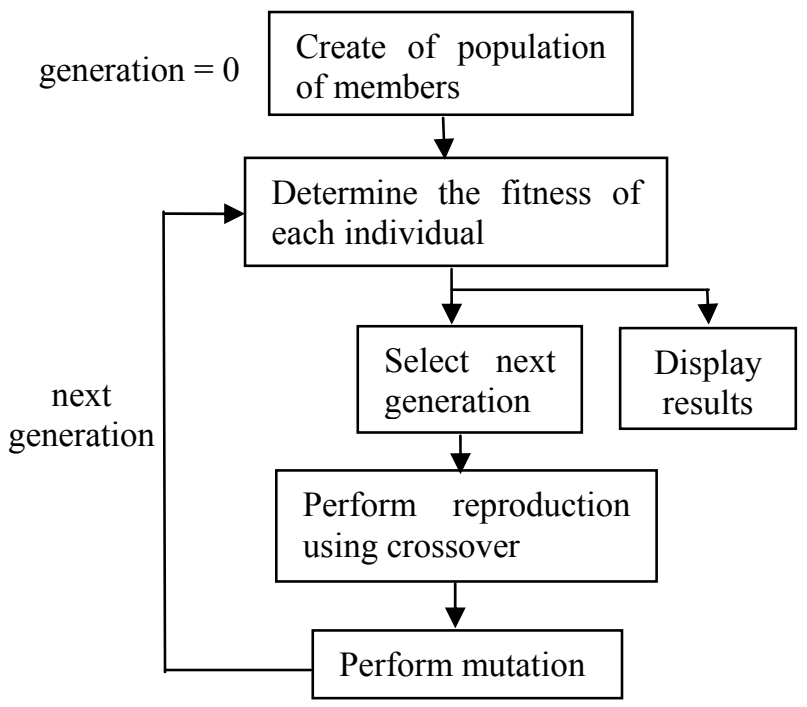

Figure 1: $\quad$ Flowchart of genetic algorithm.

In this paper a variant of the genetic method is applied for minimization of the objective function $\mathrm{E}$ of $\mathrm{N}$ parameters $\mathrm{P}_{\mathrm{i}}$ :

$$
E=f\left(P_{1}, P_{2}, \ldots, P_{N}\right)
$$

That means that values of parameters $P_{i}$ have to be chosen in such a way that the objective function $\mathrm{E}$ reaches the global minimum.

The applied variant of genetic algorithm can be described by the following steps. 
Initial population The initial population (first iteration population) is typically randomly generated i.e. every member of the initial population is combination of randomly chosen parameters:

$$
E_{j}^{1}=f\left(P_{1, j}^{1}, P_{2, j}^{1}, \ldots P_{N, j}^{1}\right), j=1,2, \ldots, N_{p o p}
$$

Each member of $\mathrm{E}_{\mathrm{j}}^{1}$ parameters $\mathrm{P}_{\mathrm{ij}}^{1}$ may be chosen in two ways.

According to the first the random value is generated using Gaussian distribution with variance $\sigma$ and mean equals to the given value of the parameter in the zero iteration $\mathrm{P}^{0}{ }_{\mathrm{i}}$ :

$$
\mathrm{P}_{\mathrm{i}, \mathrm{j}}^{1}=\mathrm{P}_{\mathrm{i}}^{0} \pm \sqrt{-2 \sigma^{2}\left(\mathrm{P}_{\mathrm{i}, \max }-\mathrm{P}_{\mathrm{i}, \min }\right) \ln \mathrm{R}}
$$

where $\mathrm{R}$ is a random number between 0 and 1 . Maximum and minimum values of parameters $\mathrm{P}_{\mathrm{i}, \max }$ and $\mathrm{P}_{\mathrm{i}, \text { min }}$ are defined in advance depending of considered problem.

In the second way uniform distribution is used in the interval $\left[\mathrm{P}_{\mathrm{i}, \max }-\mathrm{P}_{\mathrm{i}, \min }\right]$ :

$$
\mathrm{P}_{\mathrm{i}, \mathrm{j}}^{1}=(1-\mathrm{R}) \mathrm{P}_{\mathrm{i}, \min }+\mathrm{RP} \mathrm{P}_{\mathrm{i}, \max }
$$

In this work the second approach is used, which enables faster convergence when the initial solution is far from final one.

Selection of parents The selection of parents also may be performed in different ways. In this work, the members of one population are sorted from the worst to the best one depending of values of the function E. The best member of the population has the smallest value of the function E. Then, father and mother are defined by random choice. In addition, in order to provide higher probability of choice for parents with smaller values of E, random numbers are reduced from the smallest to the largest one by special procedure.

Reproduction Using the method of crossover a new child is created, which typically shares many of the characteristics of its parents. The new child may be simple arithmetic mean of its parents. In this work the weighted mean is used and weight is randomly defined:

$$
\mathrm{P}_{\mathrm{i}, \text { child }}=(1-\mathrm{R}) \mathrm{P}_{\mathrm{i} \text {,father }}+\mathrm{RP}_{\mathrm{i}, \text { mother }}
$$

The value of the random number $\mathrm{R}$ in eqn (6) in classical problems usually ranges from 0 to 1 , but in this case $\mathrm{R}$ ranges from -1 to 2 to provide wider range of combinations of features from parents.

Mutations The algorithm allows for a small chance of mutation with probability defined in advance. Parameters of new child are chosen randomly in the range $\left[\mathrm{P}_{\mathrm{i}, \min }-\mathrm{P}_{\mathrm{i}, \max }\right]$. 
Elitism The new member is generated by presented processes of selection of parents, reproduction and mutations. This member survives if it is better than the worst member in the parent's generation. The procedure repeats until $\mathrm{N}_{\text {bred }}$ new survived members are produced. $N_{\text {bred }}\left(\leq N_{\text {pop }}\right)$ is defined number depending of considered problem. The new generation is created when $\mathrm{N}_{\text {bred }}$ members of the previous generation is replaced with $\mathrm{N}_{\text {bred }}$ members of the new generation. The procedure repeats from generation to generation until minimum of objective function $\mathrm{E}$ or maximum number of iterations is reached.

\section{The heat conduction model}

The one-dimensional transient heat conduction through a plane slab is considered, fig. 2. The left side of the slab $(x=0)$ is isolated and the free surface $(\mathrm{x}=1)$ is exposed to the unknown heat flux $\mathrm{q}_{\mathrm{p}}(\tau)$.

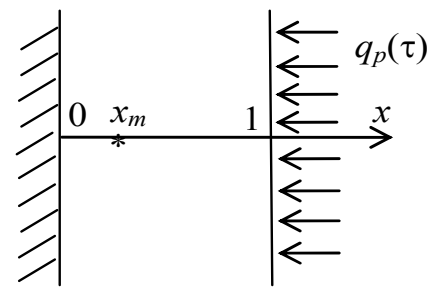

Figure 2: Heat conduction through a plane slab.

The mathematical formulation of this problem in dimensionless form is given by:

$$
\begin{gathered}
\frac{\partial \mathrm{T}(\mathrm{x}, \tau)}{\partial \tau}=\frac{\partial^{2} \mathrm{~T}(\mathrm{x}, \tau)}{\partial \mathrm{x}^{2}}, \quad 0<x<1, \tau>0, \\
\frac{\partial \mathrm{T}(0, \tau)}{\partial \mathrm{x}}=0, \quad x=0, \tau>0, \\
\frac{\partial \mathrm{T}(1, \tau)}{\partial \mathrm{x}}=\mathrm{q}_{\mathrm{p}}(\tau), \quad x=1, \tau>0, \\
T(x, 0)=0, \quad \tau=0,0 \leq x \leq 1 .
\end{gathered}
$$

On the bases on solution of similar problem [3] and introducing certain transformations analytical solution of presented problem is obtained:

$$
T(x, \tau)=\int_{0}^{\tau} q_{p}(\tau) d \tau+2 \sum_{m=1}^{\infty} e^{-\left(\frac{\beta_{m}}{2}\right)^{2} \tau} \cos \left(\frac{\beta_{m}}{2} x\right) \cos \left(\frac{\beta_{m}}{2}\right) \int_{0}^{\tau} q_{p}(\tau) e^{\left(\frac{\beta_{m}}{2}\right)^{2} \tau} d \tau
$$

where $\beta_{\mathrm{m}}=\mathrm{m} \pi$. 
It can be seen that this analytical solution is dependent of temperature flux so, $\mathbf{T}(\mathbf{P})$ is defined.

In this work transient measured temperatures $\mathbf{Y}^{\mathrm{T}}$ at the location $\mathrm{X}_{\mathrm{m}}$ are obtained by simulated experiment i.e. from solution of the heat conduction problem, eqn (11), defined by eqns (7)-(10).

\section{Results}

\subsection{Constant heat flux example}

This example considers constant heat flux (unknown parameter) applied at the free surface of the plane slab. The dimensionless value of the heat flux is $\mathrm{q}_{\mathrm{p}}=1$. A simulated temperature sensor is placed at the isolated surface, $x_{m}=0$.

For this case, temperature response $\mathrm{Y}\left(0, \tau_{\mathrm{i}}\right), \mathrm{i}=1, \ldots$,imax obtained from eqn (11), is shown, fig. 3 . In the time interval from 0 to 2 , imax $(=501)$ simulated experimental values $\mathrm{Y}_{\mathrm{i}}$ are obtained. In order to analyze influence of measurement errors, the numerical experiment in whish random distributed error with $\sigma=0.025 \mathrm{~T}_{\max }=0.05$ is added to the exact solution. It is significant error value for this type of problems.

The problem is to estimate value of $\mathrm{q}_{\mathrm{p}}$ on the basis of "measured" temperatures by minimization the objective function defined as the sum of the squared errors between model-predicted value and corresponding "experimental" data value, RMS. The problem has been solved using previously described genetic algorithm. The solution has been searched in the interval $-100 \leq q_{p} \leq 100$, with initial value $q_{p 0}=-100$.

In fig. 4, the various convergence histories are presented. Figs from $3 \mathrm{a}$ to $3 \mathrm{~h}$ represent different convergence histories until value RMS $=0.001$ is achieved. It means that the numerical value of the heat flux is estimated with error less than $0.1 \%$. These figures represent change of RMS with number of objective function calls. In this example it takes $7.3 \mathrm{~s}$ of CPU time on Pentium $4(3.06 \mathrm{GHz})$, for 100 calls. The figures also represent the influence of the number of population

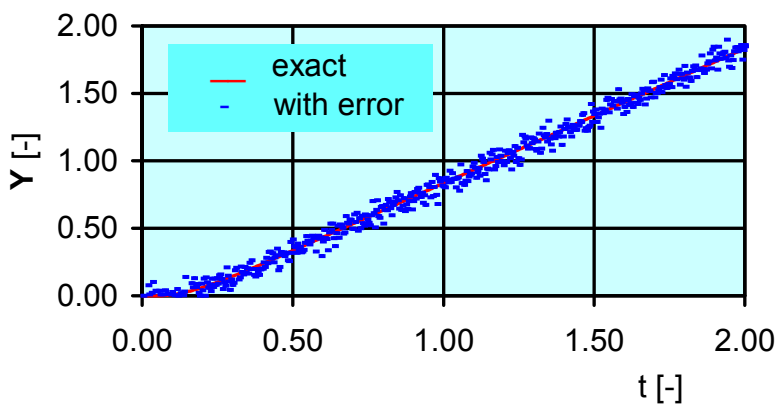

Figure 3: $\quad$ Transient temperature response $\mathrm{Y}\left(0, \tau_{\mathrm{i}}\right), \mathrm{i}=1, \ldots, 501$, for $\mathrm{q}_{\mathrm{p}}=1$. 

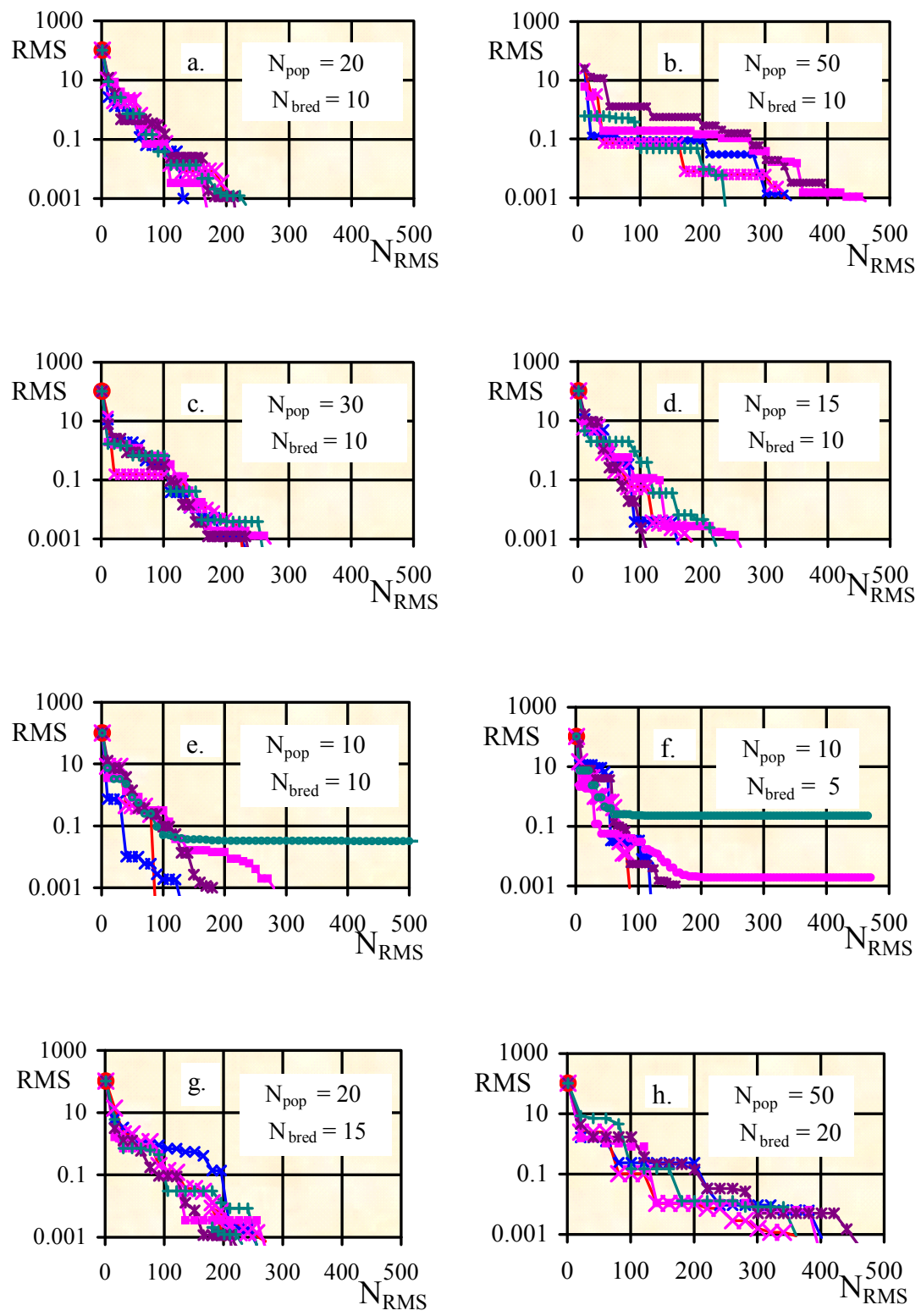

Figure 4: $\quad$ RMS change depending of the number of objective function calls $\mathrm{N}_{\mathrm{RMS}}$, different numbers of population members, $\mathrm{N}_{\text {pop }}$, and number of generated members in each generation $\mathrm{N}_{\text {bred }}$ for case $\mathrm{q}_{\mathrm{p}}=1$ and $\sigma=0$. 
members, $\mathrm{N}_{\mathrm{pop}}$, and the number of new generated members in each iteration, $\mathrm{N}_{\text {bred, }}$, to the convergence speed. Because of the statistical nature of the algorithm, each case has been run several times. The most rapid convergence has been obtained for $\mathrm{N}_{\text {pop }}=20$ and $\mathrm{N}_{\text {bred }}=10$, fig. 4a. If the number of population members $\mathrm{N}_{\text {pop }}$ is changed to $50,30,15$ and 10 , remaining $\mathrm{N}_{\text {bred }}=10$, as it is represented in fig. 4 , from $4 \mathrm{~b}$ to $4 \mathrm{e}$, respectively, the slower convergence is obtained. It can be seen that in the case with $\mathrm{N}_{\text {pop }}=10$ and $\mathrm{N}_{\text {bred }}=10$, fig. $4 \mathrm{e}$, certain runs have not solution. Similar case is with $\mathrm{N}_{\text {pop }}=10$ and $\mathrm{N}_{\text {bred }}=5$, fig. 4f. It leads to conclusion that, in considered problem here, population of 10 members is not big enough to provide new generation diversity and make progress. Fig. $4 \mathrm{~g}$ and fig. $4 \mathrm{~h}$ represent combinations with 20 and 50 members with different $\mathrm{N}_{\text {bred }}$ in respect to previous combinations. This does not lead to improvement in regard to combination with $\mathrm{N}_{\text {pop }}=20$ and $\mathrm{N}_{\text {bred }}=10$. It can be noticed that with increasing the number of the population members, the slower convergence history is obtained, fig. $4 \mathrm{~b}$ and fig. $4 \mathrm{~h}$.

The convergence history in the case with measurement errors is given in fig. 5. It can be seen that, very fast, for about 150-200 objective function calls, RMS $=\sigma=0.050$ is obtained. Error in the heat flux estimation in this case is less than $0.1 \%$.

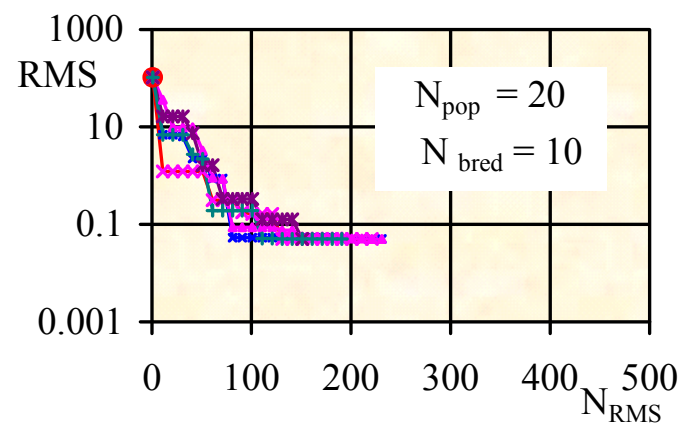

Figure 5: RMS change with objective function calls in the case $\mathrm{q}_{\mathrm{p}}=1$ and $\sigma=$ 0.050 .

\subsection{Transient heat flux example}

In this example the heat flux changes in time according to the following exponential relation:

$$
\mathrm{q}_{\mathrm{p}}(\tau)=\mathrm{a} \mathrm{e}^{-\mathrm{b} \tau}
$$

where $a$ and $b$ are unknown parameters need to be estimated.

As in the previous case, transient heat flux is determined on the basis of the response of temperature sensor at the location $x_{m}=0$, fig. 2 . In the same way, temperature response is obtained using simulated experiment i.e. from eqn (11) with inserted function for transient heat flux eqn (12). 101 temperature values 


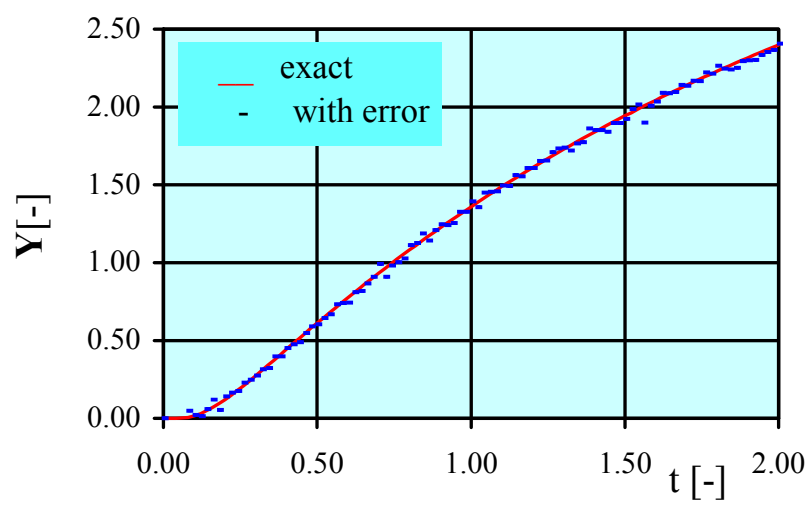

Figure 6: Transient temperature response, $\mathrm{T}\left(0, \tau_{\mathrm{i}}\right), \mathrm{i}=1, \ldots, 101$ in the case of exponential heat flux change, without and with measurement error $(\sigma=0.025)$.

have been calculated in the time interval from 0 to 2 . In order to simulate real experiment, random distributed error is added to the exact solution values with $\sigma$ $=0.014 \mathrm{~T}_{\max }=0,025$, fig. 6 .

Fig. 7 represents convergence history. The solution is obtained for about 4000 objective function calls. In this case unknown parameters $a$ and $b$ can be calculated with error less than $1 \%$ and $3 \%$ respectively. In this example it takes $1.9 \mathrm{~s}$ of CPU time on Pentium 4 (3.06 GHz), for 100 calls.

Model estimated and exact values of the heat flux are compared in fig. 8 .

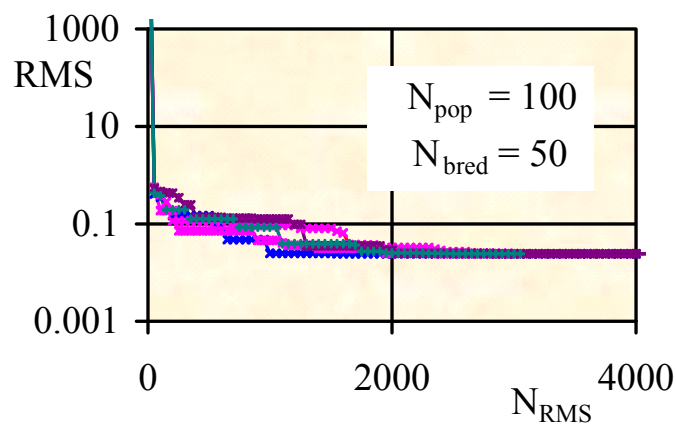

Figure 7: $\quad$ RMS change depending of the number of objective function calls $\mathrm{N}_{\mathrm{RMS}}$, for exponential form of heat flux function and measurement error with $\sigma=0.025$. 


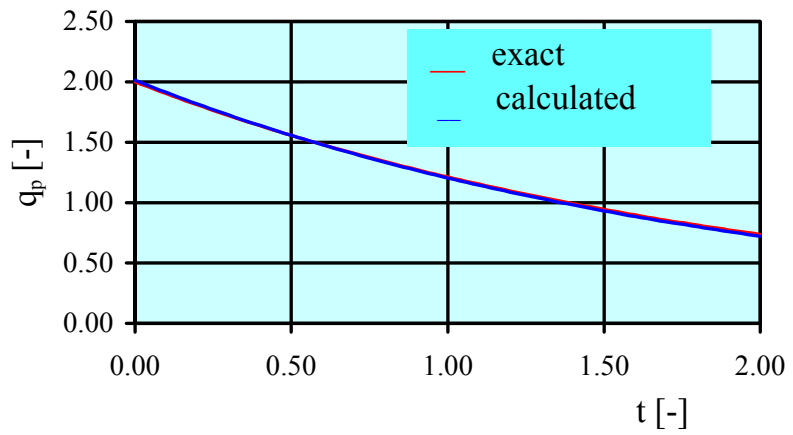

Figure 8: Comparison between estimated heat flux change in the case of exponential flux function and measurement temperature error with $\sigma=0.025$, and exact solution.

Fig. 9 represents comparison between the temperature calculations with exact parameters and with estimated parameters $\mathrm{a}$ and $\mathrm{b}$ of the heat flux exponential function.

Increasing measurement error to $\sigma=0.050$ leads to parameter estimation error for a and $\mathrm{b}$ of $1 \%$ and $5 \%$ respectively.

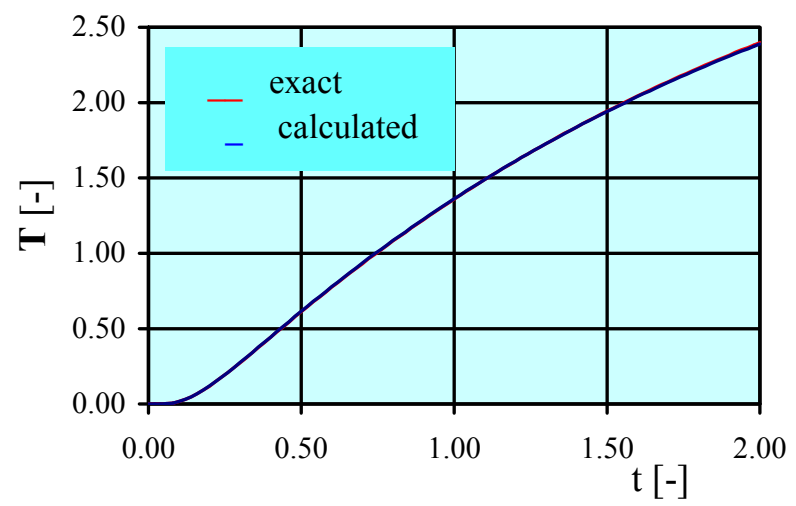

Figure 9: Calculation of transient temperature response $\mathrm{T}\left(0, \tau_{\mathrm{i}}\right)$ for the case of exponential shape of the heat function with error $(\sigma=0.025)$ in compare to exact solution.

\section{Conclusion}

In this paper the application of the genetic algorithm to the heat flux estimation on the bases of transient temperature response is presented and analyzed. The 
case of constant heat flux (one unknown parameter) and case of exponential transient heat flux (two parameters) are analyzed. The convergence history dependences of different combinations of population and breed number are presented. It can be concluded that the genetic algorithm may be successfully used in both cases. The constant heat flux can be estimated with high accuracy even if measurement is realized with relatively significant error. In the case of transient heat flux, estimation depends of the measurement error.

\section{References}

[1] Kanevce, G.H. Kanevce, L. P., Mitrevski, V.B., Dulikravich, G. S. \& Orlande, H.R.B., Inverse approaches to drying of thin bodies with significant shrinkage effects, Journal of heat transfer - transactions of the ASME, 129(3), pp. 379-386, 2007.

[2] Beck, J. V. \& Arnold, K. J., Parameter Estimation in Engineering and Science, John Wiley \& Sons, Inc., New York, p. 370, 1977.

[3] Ozisik M. N. \& Orlande H. R. B., Inverse Heat Transfer: Fundamentals and Applications, Taylor and Francis, New York, 2000.

[4] Woodbury K. A., Application of Genetic Algorithms and Neural Networks to the Solution of Inverse Heat Conduction Problems, 4th International Conference on Inverse Problems in Engineering, Ed. H. R. B. Orlande, epapers, Rio de Janeiro, pp. 73-88, 2002.

[5] Mitchell, Melanie, An introduction to Genetic Algorithms, MIT Press, 1999, pp. 2-11, 1999.

[6] Adam Marczyk, Genetic Algorithms and Evolutionary Computation, What is a genetic algorithm, Copyright (C) 2004. http://www.talkorigins.org/faqs /genalg/genalg.html 